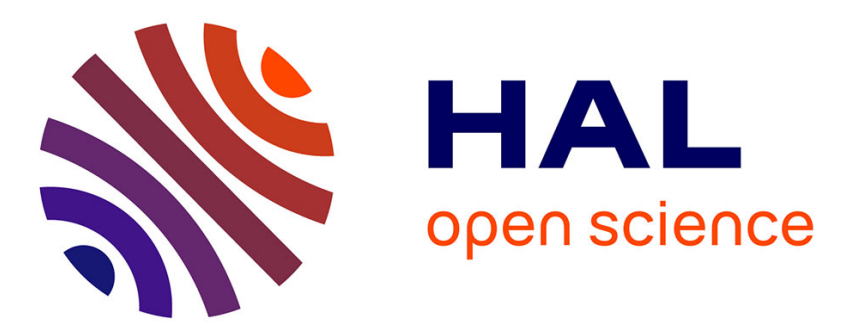

\title{
A classification of elementary particles in $d=4$ following a simple geometrical hypothesis in real space
}

Nathalie Olivi-Tran, Nicolas Gottiniaux

\section{To cite this version:}

Nathalie Olivi-Tran, Nicolas Gottiniaux. A classification of elementary particles in $\mathrm{d}=4$ following a simple geometrical hypothesis in real space. Advanced Studies in Theoretical Physics, 2013, 7 (18), pp.853 - 857. 10.12988/astp.2013.3778 . hal-00853870

\section{HAL Id: hal-00853870 \\ https://hal.science/hal-00853870}

Submitted on 24 Aug 2013

HAL is a multi-disciplinary open access archive for the deposit and dissemination of scientific research documents, whether they are published or not. The documents may come from teaching and research institutions in France or abroad, or from public or private research centers.
L'archive ouverte pluridisciplinaire HAL, est destinée au dépôt et à la diffusion de documents scientifiques de niveau recherche, publiés ou non, émanant des établissements d'enseignement et de recherche français ou étrangers, des laboratoires publics ou privés. 


\title{
A classification of elementary particles in $\mathrm{d}=4$ following a simple geometrical hypothesis in real space
}

\author{
Nathalie Olivi-Tran* and Nicolas Gottiniaux \\ Laboratoire Charles Coulomb, CNRS, \\ UMR 5221, place Eugene Bataillon, \\ 34095 Montpellier cedex 5, France, \\ Laboratoire Charles Coulomb, \\ Universite Montpellier 2, \\ place Eugene Bataillon, \\ 34095 Montpellier cedex 5, France \\ * corresponding author \\ email: Nathalie.Olivi-Tran@univ-montp2.fr
}

(Dated: July 12, 2013)

\begin{abstract}
We made the hypothesis that our universe is fourdimensional: time $t$ is treated as a 'regular' dimension. So in the $(t, x, y, z)$ coordinates system, we present a simple approach of all the elementary particles which might be four,three and two-dimensional. This hypothesis leads to a unified approach of all particles.
\end{abstract}

Keywords: Grand Unified Theory; fourdimensional space; real space theory 


\section{INTRODUCTION}

All the theories which aim to understand the elementary particles treat time $t$ as a special dimension. Thus, many physicists deal with $n+1$ space dimensions in particle physics, where the +1 correspond to the 'special' temporal dimension and thus treated differently. As previously published [1-3], time may be seen as a function of space dimension, if our threedimensional universe is embedded in a fourdimensional space (due to the curvature of our threedimensional universe). So, here we will present a simple hypothesis about the classification of elementary particles based on the fact that the space is fourdimensional and that time $t$ is a dimension like $x, y$ and $z$. We will not make the calculations to prove that our hypothesis fits the Standard Model and the experimental results. Here, the whole paper is dedicated to our hypothesis. Indeed, with simple arguments, it seems to lead to the GUT.

\section{THEORY}

I make the assumption that our threedimensional universe is embedded in a four dimensional euclidean space. Time is a function of the fourth dimension of this space [1-3]. If we apply this hypothesis to particle physics, we may say that elementary particles are fourdimensional, threedimensional and twodimensional.

The coordinates $(x, y, z, t)$ are not orthonormal.Indeed, time $t$ evolves as $\log (r)$ where $r$ is the comoving distance in cosmology[1].

Let us make the additional assumption that for each of these four dimensions there are functions like $\exp \left(i k r_{j}\right)$ with $\left(r_{j}=x, y, z, t\right)$ which vibrate (like in string theory). So, our reasoning is simply the description of how to distribute these functions in the fourdimensional space. In the following, the reasoning applies in real space.

A previous paper of mine (see [4]) predicts that the Higgs potential in real space is a hypercubic box in our fourdimensional space.

To obtain the first family of fermions from the Standard Model ( i.e. quark up, quark down, electron, electron neutrino) one may say that:

- the electron is fourdimensional $(t, x, y, z)$

- the two quarks are three dimensional $(t, x, y)$ and $(t, x, z)$ : to make the difference between these two quarks one has to use the Fermi Dirac equations. Indeed it is only 
when these quarks propagate that one can make a difference between them: the Dirac matrices imply an exchange (infinitesimal rotations) between $t, x$ and $y$ and $t, x, z$ which are not equivalent for the two quarks (due to the irreversibility of time which is due to the expansion of the universe-[1-3] ).

- finally, the electronic neutrino is twodimensional $(t, x)$ and $x, y$ and $z$ are equivalent. It is only due to the Fermi Dirac equation that when this neutrino propagates, there are infinitesimal rotations between the characteristic coordinates (leading to flavor oscillations).

To obtain the remaining fermions (elementary particles), one has to modify the quantum number $n$ (similar to the quantum number during oscillation in a hypercubic box, due to my Higgs potential [4]). Thus, the remaining fermions of the Standard Model may be seen as excited states of the first fermion family.

Bosons may be classified with the same assumptions:

- the photon is twodimensional $(x, y)$ but has no temporal $t$ coordinate -no mass(indeed with my Higgs potential [4], time at square is proportional to the mass).

- the gluon is three dimensional $(x, y, z)$ and has no temporal dimension- no mass. (during the strong interaction, one gluon interferes (positive interferences) with two quarks: $x$ on $x, y$ on $y$ etc.)

- the $\mathrm{Z}$ and $\mathrm{W}$ bosons are threedimensional with mass $(t, x, y)$ (at high energies, the coordinate $t$ (time) tends to zero leading to a photon $(x, y)$ )

- the Higgs boson is fourdimensional [4]

In all this description, one has to separate the geometrical description of particles from their equation of propagation.

This is the hypothesis for a new classification of elementary particles. Up to now, all calculations have been made in the space of functions, leading to symmetries which are difficult to unify. But what we can say is that the symmetries in the Standard Model result from the dimensions of the elementary particles and the way they propagate in a space of functions, so in our opinion, the symmetries do not give the entire description of elementary particles. 


\section{CONCLUSION}

This paper showed that with simple geometrical assumptions in real space (fourdimensional space), one may describe all the elementary particles. There is much work to do to prove that this hypothesis fits the experimental results. This will be done step by step and published later. But, at first, we have to study the relation between time $t$ and the mass of elementary particles: my previous paper on the Higgs field [4] showed that $M=T^{2} / L^{2}$ where $M$ is the mass $T$ the characteristic time and $L$ a characteristic length (obtained by a dimensional analysis). So, mass is not related to the 'volume' of the particle. What is mass (i.e. rest mass)?

Acknowledgments: the authors acknowledge interesting discussions with R.GottiniauxTran

[1] N.Olivi-Tran and P.M.Gauthier, The FLRW cosmological model revisited: Relation on the local time with the local curvature and consequences on the Heisenberg uncertainty principle Adv. Studies Theor. Phys. vol.2 no 6 (2008) 267-270

[2] N.Olivi-Tran What if our three dimensional curved universe was embedded in four dimensional space? Consequences on the EPR paradox Adv. Studies Theor. Phys., Vol. 3, (2009), no. 12, $489-492$

[3] N.Olivi-Tran Dimensional analysis of Einstein's fields equations Adv. Studies Theor. Phys., Vol. 3, (2009), no. 1, 9 - 12

[4] N.Olivi-Tran Is it the Higgs scalar field? Advanced Studies in Theoretical Physics Vol. 4, 2010, no. $13,633-636$ 